\title{
Picomole per Liter per Kilogram per Meter Squared
}

National Cancer Institute

\section{Source}

National Cancer Institute. Picomole per Liter per Kilogram per Meter Squared. NCI

Thesaurus. Code C119485.

A unit of concentration equal to picomoles per liter, divided by kilograms per meter squared. 